are dense and impermeable, and are free of other disintegrative agents. In this range of cements, airentrainment would undoubtedly be beneficial, and good workmanship would be absolutely necessary.

3. A cement having a sulfate reaction value of over 65 should be considered as relatively nonresistant to sulfate attack; the higher that value, the lower the resistivity of the cement. Concrete or mortar structures made with such a cement may be expected to have a relatively short life when exposed to sulfate waters, regardless of the quality of the workmanship or the presence of beneficial agents.

The authors take pleasure in acknowledging the contribution of I. C. Bechtold in the development of the sulfate susceptibility test, and the assistance of A. C. Bonanno, E. G. Siggers, and C. Pinkerton in numerous analyses and tests.

\section{References}

[1] Am. Soc. Testing Materials, Standard methods of chemical analysis of portland cement, C 114-47.

[2] P. H. Bates, A. J. Phillips, and R. J. Wig, Tech. Pap. BS 2, 7 (1912) T12.
[3] R. H. Gogue, Chemistry of portland cement, pp. 508 to 520 (Reinhold Pub. Co., New York, N. Y., 1947).

[4] R. H. Bogue, Studies on the volume stability of portland cement pastes, privately printed by the Portland Cement Association Fellowship, PCAF Paper 55 (1949).

[5] R. H. Bogue, Wm. Lerch, and W. C. Taylor, Ind. Eng. Chem. 26, 1049 (1934); PCAF Paper 28.

[6] F. K. Cameron and J. W. Bell, U. S. Dept. Agr. Bul. 23, 47 (1906)

[7] G R. Gause, Am. Soc. Testing Materials Bul. 112, 17 (1941).

[8] W. C. Hansen and E. E. Pressler, Ind. Eng. Chem. 39, 1280 (1947)

[9] W. C. Hansen, personal communication.

[10] Wm. Lerch, Proc. Am. Soc. Testing Materials 46, 1252 (1946).

[11] Wm. Lerch, F. W. Ashton, and R. H. Bogue, BS J. Research 2, 715 (1929) RP54; PCAF Paper 19.

[12] D. G. Miller, Report of Working Committee on Sulfate Resistance, Com. C-1, ASTM (July 1943).

[13] D. G. Miller and P. W. Manson, U. S. Dept. Agr. Bul. 358; Proc. Am. Soc. Testing Materials 40, 988 (1940).

[14] M. A. Swayze, Am. J. Sci. 244, 1, 65 (1946).

[15] T. Thorvaldson, R. H. Harris, and D. Wolochow, Ind. Eng. Chem. 17, 467 (1925).

[16] A. H. White and H. S. Kemp, Concrete 51, 105 (1943).

Washington, June 17, 1949.

\title{
Cascade-Connected Attenuators
}

\author{
By Robert W. Beatty
}

\begin{abstract}
A method is presented for evaluating the error obtained when the attenuation of a number of cascade-connected attenuators is determined by adding the attenuation of each unit. The error is caused by mismatches at the attenuator junctions and is expressed in terms of reflection coefficients measured at the junctions. The analysis is pertinent to the case in which individual calibrated attenuators are available, but it is not feasible or possible to calibrate the combination of two or more attenuators. A nomogram is drawn that shows the limits of error (for two cascaded attenuators) in terms of voltage standing-wave ratios. Inspection of the nomogram shows that the error for typical UHF and microwave attenuators is generally of the same order of magnitude as the calibration errors.
\end{abstract}

\section{Introduction}

In order to obtain a desired attenuation, it is often necessary to connect two or more calibrated attenuators in series (cascade). Cascade-connected attenuators are used as "gage blocks" to extend the range of power meters without appreciable loss of accuracy. ${ }^{1}$ Fixed and variable attenuators are cascade-connected in order to obtain increased range.

The attenuation of such combinations could be obtained by accurate measurement (calibration), but this is not feasible or possible in many cases because attenuator calibration equipment is not widely available.

The total attenuation of cascade-connected attenuators is usually obtained by adding the attenuation

1 C. G. Montgomery, Technique of microwave measurements, p. 835 (McGrawHill Book, Co., Inc., New York, N. Y., 1947). of each unit. If mismatch exists at the attenuator junctions, the attenuation obtained by this method is incorrect. The resulting error is a function of the amount of mismatch and may be evaluated in terms of the reflection coefficients measured at the attenuator junctions.

\section{Analysis}

In the following analysis, UHF or microwave attenuators are considered. It is assumed that the individual attenuators have been calibrated in a transmission-line system having the same characteristic impedance $\left(\dot{Z}_{0}\right)$ and critical dimensions as the system in which the attenuators are to be used. A further requirement is that the attenuators are passive linear four-terminal networks having terminals that permit connection to the transmissionline system without discontinuity. 

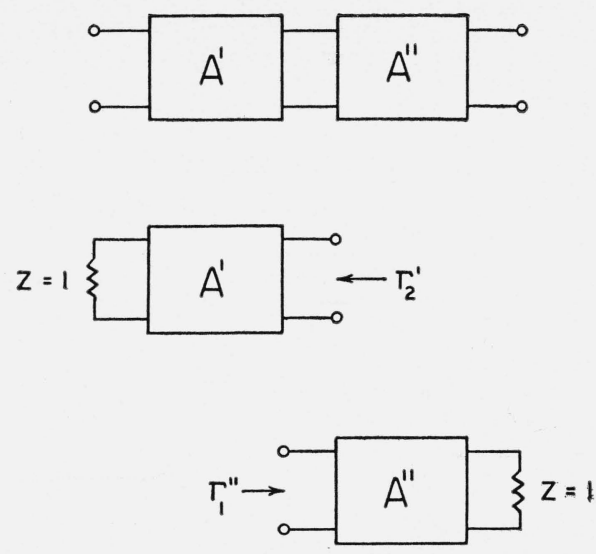

$$
A_{T}=A_{T}^{\prime}+A_{T}^{\prime \prime}+20 \log _{10}\left|1-\Gamma_{2}^{\prime} \Gamma_{1}^{\prime \prime}\right| d b
$$

Figure 1. Two cascaded networks.

For convenience, wave matrices ${ }^{2}$ are used and the following symbols are defined:

$\Gamma_{1}=$ Voltage reflection coefficient measured at the input terminals of an attenuator when the output terminals are connected to an impedance $Z_{0}$.

$\Gamma_{2}=$ Voltage reflection coefficient measured at the output terminals of an attenuator when the input terminals are connected to an impedance $Z_{0}$

$Z=$ Voltage transmission coefficient of an attenuator terminated in an impedance $Z_{0}$.

The elements of the wave matrix designated as the $A$-matrix are:

$$
\left.\begin{array}{ll}
A_{11}=\frac{1}{T} & A_{12}=-\frac{\Gamma_{2}}{T} \\
A_{21}=\frac{\Gamma_{1}}{T} & A_{22}=T-\frac{\Gamma_{1} \Gamma_{2}}{T}
\end{array}\right\}
$$

The attenuation of two cascaded attenuators is considered first. This is followed by the case of three attenuators and finally, the attenuation of any number of attenuators is obtained.

\section{Two Attenuators}

The cascade connection of two attenuators is shown in figure 1 . The $A$-matrix of the combination is:

$A=\left[\begin{array}{ll}A_{11} & A_{12} \\ A_{21} & A_{22}\end{array}\right]=\left[\begin{array}{cc}A_{11}^{\prime} & A_{12}^{\prime} \\ A_{21}^{\prime} & A_{22}^{\prime}\end{array}\right] \times\left[\begin{array}{ll}A_{11}^{\prime \prime} & A_{12}^{\prime \prime} \\ A_{21}^{\prime \prime} & A_{22}^{\prime \prime}\end{array}\right]$

${ }^{2}$ George L. Ragan, Microwave transmission circuits, p. 551 to 554 (McGraw Hill Book Co., Inc., New York, N. Y., 1948).
This is equivalent to,

$$
A=\left[\begin{array}{cc}
A_{11}^{\prime} A_{11}^{\prime \prime}+A_{12}^{\prime} A_{21}^{\prime \prime} & A_{11}^{\prime} A_{12}^{\prime \prime}+A_{12}^{\prime} A_{22}^{\prime \prime} \\
A_{21}^{\prime} A_{11}^{\prime \prime}+A_{22}^{\prime} A_{21}^{\prime \prime} & A_{21}^{\prime} A_{12}^{\prime \prime}+A_{22}^{\prime} A_{22}^{\prime \prime}
\end{array}\right]
$$

From inspection of eq 2 and 3 ,

$$
A_{11}=A_{11}^{\prime} A_{11}^{\prime \prime}+A_{12}^{\prime} A_{21}^{\prime \prime}
$$

Substituting eq 1 in eq 4

$$
\frac{1}{T}=\frac{1}{T^{\prime} T^{\prime \prime}}\left(1-\Gamma_{2}^{\prime} \Gamma_{1}^{\prime \prime}\right) .
$$

The attenuation (in decibels) of the combination is:

$$
A_{T}=20 \log _{10}\left|\frac{1}{T}\right| .
$$

The attenuation of a network ${ }^{34}$ is defined as its insertion loss when placed in a matched system. From eq 5 and 6 it follows that

$$
\begin{gathered}
A_{T^{\prime}}=20 \log _{10}\left|\frac{1}{T^{\prime}}\right|+20 \log _{10}\left|\frac{1}{T^{\prime \prime}}\right|+20 \log _{10}\left|1-\Gamma_{2}^{\prime} \Gamma_{1}^{\prime \prime}\right| . \\
A_{T}=A_{T}^{\prime}+A_{T}^{\prime \prime}+20 \log _{10}\left|1-\Gamma_{2}^{\prime} \Gamma_{1}^{\prime \prime}\right|
\end{gathered}
$$

The last ter $\mathrm{n}$ in eq 8 represents the error $\left(\epsilon_{2}\right)$. This term must be added to the sum of the attenuations of the individual units to obtain the correct attenuation for the combination.

It may be expedient in some cases to measure only the voltage standing-wave ratios $\sigma_{2}^{\prime}$ and $\sigma_{1}^{\prime \prime}$ corresponding to $\Gamma_{2}^{\prime}$ and $\Gamma_{1}^{\prime \prime}$. In this event only the magnitudes of $\Gamma_{2}^{\prime}$ and $\Gamma_{1}^{\prime \prime}$ are obtained, and the actual value of $\epsilon_{2}$ lies between the limits

$$
\begin{aligned}
& 20 \log _{10}\left(1+\frac{\sigma_{2}^{\prime}-1}{\sigma_{2}^{\prime \prime}+1} \cdot \frac{\sigma_{1}^{\prime \prime}-1}{\sigma_{1}^{\prime \prime}+1}\right)>\epsilon_{2}> \\
& 20 \log _{10}\left(1-\frac{\sigma_{2}^{\prime}-1}{\sigma_{2}^{\prime}+1} \cdot \frac{\sigma_{1}^{\prime \prime}-1}{\sigma_{1}^{\prime \prime}+1}\right) .
\end{aligned}
$$

From the measured values of $\sigma_{2}^{\prime}$ and $\sigma_{1}^{\prime \prime}$, the limits of error $\epsilon_{2}$ may be readily determined by use of the nomogram of figure 4.

\section{Three Attenuators}

The cascade connections of three attenuators are shown in figures 2 and 3 . The A-matrix of the combination is:

\footnotetext{
3 See footnote 2, pp. 550 to 552

4 See footnote 1, pp. 679 to 682 .
} 


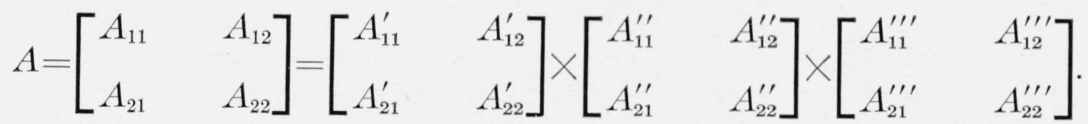
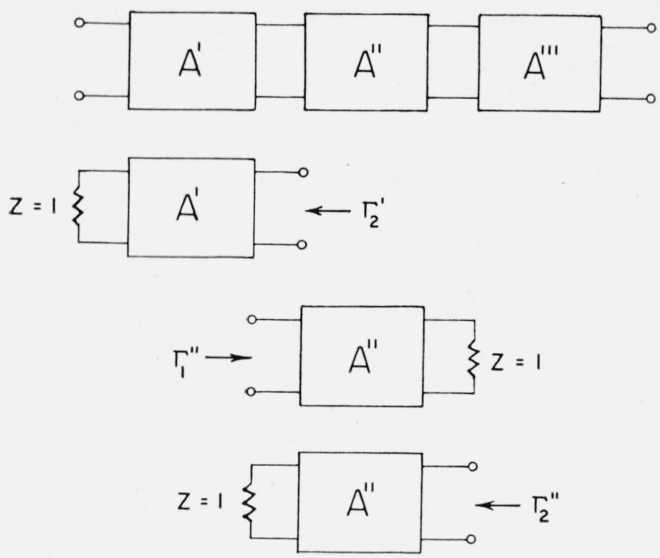

$$
A_{T}^{\prime \prime}=20 \operatorname{LOG}_{10}\left|\frac{1}{T^{\prime \prime}}\right|
$$

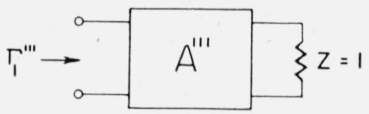

$A_{T}=A_{T}^{\prime}+A_{T}^{\prime \prime}+A_{T}^{\prime \prime \prime}+20 \operatorname{LOG}_{10}\left|\left(1-\Gamma_{2}^{\prime} \Gamma_{1}^{\prime \prime}\right)\left(1-\Gamma_{2}^{\prime \prime} \Gamma_{1}^{\prime \prime \prime}\right)-\Gamma_{2}^{\prime} \Gamma_{1}^{\prime \prime \prime}\left(T^{\prime \prime}\right)^{2}\right| d b$

FIGURE 2. Three cascaded networks (each network considered a unit).
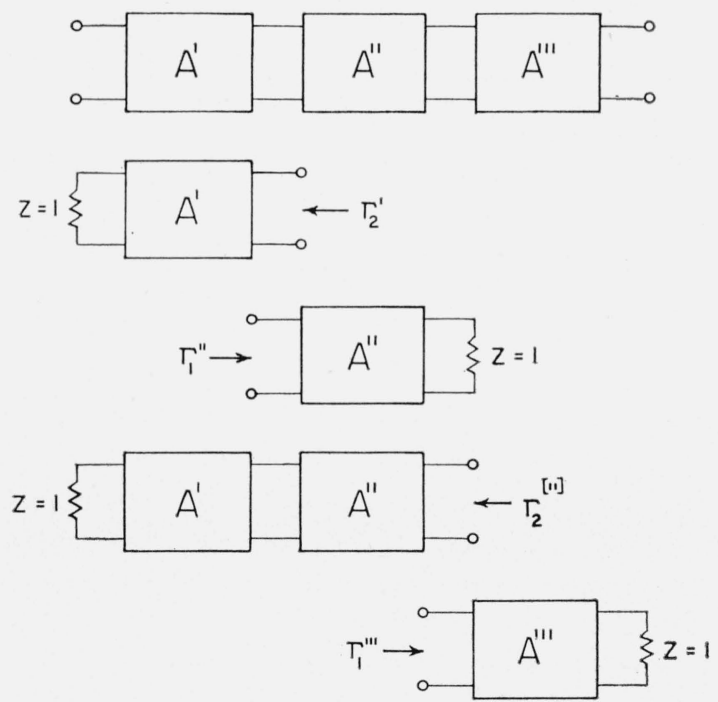

$$
\begin{aligned}
& \left(A_{T}^{\prime}\right)^{\prime \prime}=A_{T}^{\prime}+A_{T}^{\prime \prime}+20 \operatorname{LOG}_{10}\left|1-\Gamma_{2}^{\prime \prime} \Gamma_{1}^{\prime \prime}\right| d b \\
& A_{T}=\left(A_{T}^{\prime}\right)^{\prime \prime}+A_{T}^{\prime \prime \prime \prime}+20 \operatorname{LOG}_{10}\left|1-\Gamma_{2}^{[\prime \prime]} \Gamma_{1}^{\prime \prime \prime}\right| d b
\end{aligned}
$$

FIgURE 3. Three cascaded networks (two input networks considered one unit).
Only $A_{11}$ need be considered:

$$
A_{11}=A_{11}^{\prime \prime \prime}\left(A_{11}^{\prime} A_{11}^{\prime \prime}+A_{12}^{\prime} A_{21}^{\prime \prime}\right)+A_{21}^{\prime \prime \prime}\left(A_{11}^{\prime \prime} A_{12}^{\prime \prime}+A_{12}^{\prime} A_{22}^{\prime \prime}\right) \text {. }
$$

This is equivalent to:

$$
\frac{1}{T}=\frac{1}{T^{\prime} T^{\prime \prime} T^{\prime \prime \prime}}\left[\left(1-\Gamma_{2}^{\prime} \Gamma_{1}^{\prime \prime}\right)\left(1-\Gamma_{2}^{\prime \prime} \Gamma_{1}^{\prime \prime \prime}\right)-\Gamma_{2}^{\prime} \Gamma_{1}^{\prime \prime \prime}\left(T^{\prime \prime}\right)^{2}\right] .
$$

The attenuation of the combination is:

$$
\begin{aligned}
A_{T}= & A_{T}^{\prime}+A_{T}^{\prime \prime}+A_{T}^{\prime \prime \prime}+ \\
& 20 \log _{10} \mid\left(1-\Gamma_{2}^{\prime} \Gamma_{1}^{\prime \prime}\right)\left(1-\Gamma_{2}^{\prime \prime} \Gamma_{1}^{\prime \prime \prime}\right)-\Gamma_{2}^{\prime} \Gamma_{1}^{\prime \prime \prime}\left(T^{\prime \prime}\right)^{2} .
\end{aligned}
$$

The last term in eq 13 represents the error $\epsilon_{3}$. It is apparent that $\epsilon_{3}$ depends not only upon the reflection coefficients at the junctions but also upon the transmission coefficient $T^{\prime \prime}$ of the central attenuator. From eq 6 it can be seen that $\left|T^{\prime \prime}\right|$ is a function of $A_{T}^{\prime \prime}$. When the attenuation of the central unit is large, $\left|T^{\prime \prime}\right|$ is small and the following approximation is made:

$$
\epsilon_{3} \approx 20 \log _{10}\left|1-\Gamma_{2}^{\prime} \Gamma_{1}^{\prime \prime}\right|+20 \log _{10}\left|1-\Gamma_{2}^{\prime \prime} \Gamma_{1}^{\prime \prime \prime \prime \prime}\right| .
$$

The two components of $\epsilon_{3}$ can readily be obtained from the measured values of $\Gamma_{2}^{\prime}, \Gamma_{2}^{\prime \prime}, \Gamma_{1}^{\prime \prime}$, and $\Gamma_{1}^{\prime \prime \prime}$, using the nomogram of figure 4. The limits of $\epsilon_{3}$ are obtained in this case by adding the individual limits.

If the reflection coefficients are measured in a different way, it is possible to obtain the attenuation $A_{T}$ without considering $T^{\prime \prime}$. This procedure is described in the following discussion.

\section{Any Number ( $n)$ of Attenuators}

The attenuation of any number $(n)$ of cascadeconnected attenuators is:

$$
A_{T}=\sum_{K=1}^{n} A_{T}^{K}+\epsilon_{n}
$$

In the above equation the symbol $K$ is a superscript designating the position of the attenuator, numbering from the input of the cascade-connection.

In determination of $\epsilon_{n}$, it is unnecessary to consider complex transmission coefficients if the following procedure (illustrated in fig. 3) is used. First, the attenuation of the combination of the two units at the input end is determined by the method illustrated in figure 1. The two attenuators are now considered as a single unit whose attenuation is known. The attenuation of the combination of this 


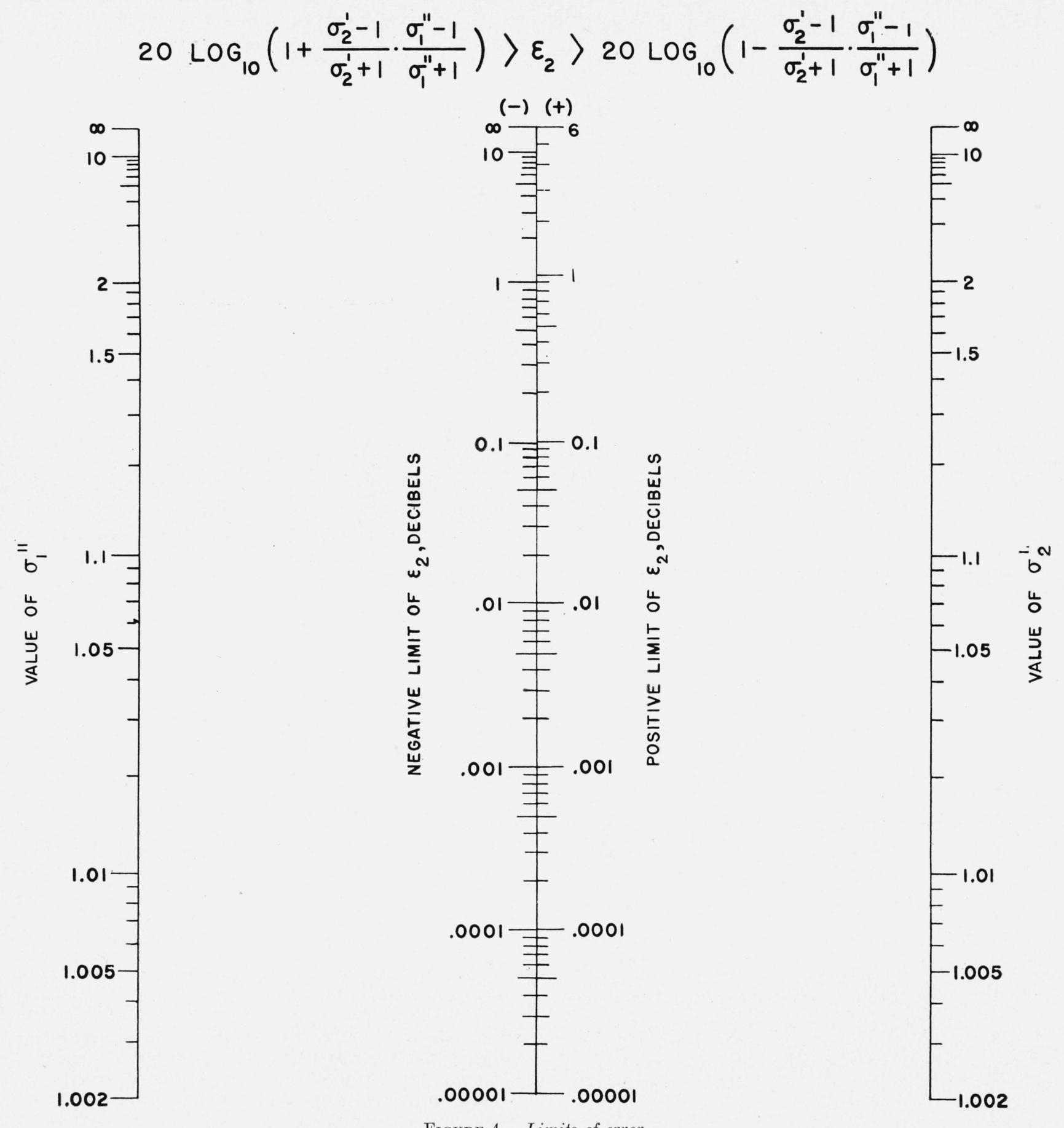

Figure 4.-Limits of error. 
unit with the third is also determined by the method illustrated in figure 1.

It is apparent that a continuation of this process will yield the correct attenuation of the combination of any number of attenuators.

An extension of the process illustrated in figure 3 yields :

$$
A_{T}=\sum_{K=1}^{n} A_{T}^{K}+\sum_{l=2}^{n} 20 \log _{10}\left|1-\Gamma_{2}^{[l-1]} \Gamma_{1}^{l}\right|
$$

where,

$A_{T}^{K}=$ attenuation of $K^{\text {th }}$ network.

$\Gamma_{1}^{l}=$ reflection coefficient measured at the input terminals of the $l^{\text {th }}$ attenuator with its output terminals connected to an impedance $Z_{0}$.

$$
\begin{aligned}
\Gamma_{2}^{[l-1]}= & \text { reflection coefficient measured at the } \\
& \text { output terminals of the }[l-1]^{t h} \text { at- } \\
& \text { tenuator with the input terminals of } \\
& \text { attenuator no. } 1 \text { connected to an } \\
& \text { impedance } Z_{0} .
\end{aligned}
$$

These results may be applied to attenuators that are not used in a transmission-line system if expressions

of the form $\left(\frac{Z-Z_{0}}{Z+Z_{0}}\right)$ are substituted for the appropriate reflection coefficients and the same reference impedance $\left(Z_{0}\right)$ is used throughout.

Washington, May 23, 1950.

\title{
Total Ionization of Hydrocarbons From Mass Spectral Data
}

\author{
By Fred L. Mohler, Laura Williamson, and Helen M. Dean
}

\begin{abstract}
The total ionization is computed by adding all the mass peaks in the spectrum and multiplying by the sensitivity (current per unit pressure) for the maximum peak. This is divided by the corresponding product for $n$-butane to obtain a relative value independent of the units used. Data are taken from the API Catalog of Mass Spectral Data using revised values of sensitivity obtained by measuring pressure with a micromanometer. Total ionizations of all the isomers of a compound are nearly equal with only a few values differing greatly from the mean value. This is true in all cases where data are available for many isomers. With some exceptions total ionization increases with increasing number of carbon atoms in each series $\mathrm{C}_{n} \mathrm{H}_{2 \mathrm{n}+2}, \mathrm{C}_{\mathrm{n}} \mathrm{H}_{2 \mathrm{n}}$, etc. Total ionization tends to decrease in compounds with the same number of carbon atoms and decreasing number of hydrogen atoms but there are many exceptions. Tables summarize data for 198 hydrocarbons.

Values of total ionization of isomers will in some cases permit computing all isomers as a group in chemical analysis without identifying the compounds.
\end{abstract}

\section{Introduction}

Tables of mass spectra [1] ${ }^{1}$ commonly give the intensity of mass peaks relative to the maximum peak taken as 100 and the sensitivity (current per unit pressure in the gas reservoir) for the maximum peak in arbitrary units. The tables also give the sensitivity for the maximum peak of $n$-butane at mass 43 in the same units. The ratio of the sensitivity of any compound to that of $n$-butane is a number that is independent of the arbitrary units of current and rate of leak from the gas reservoir to the ionization chamber. The sum of all the mass peaks times the sensitivity for the maximum peak is a number proportional to the total ionization for the compound, and the ratio of this to the total ionization of $n$-butane is again a number independent of the arbitrary units. These relative values will depend to some extent on instrumental selectivity and will differ somewhat for different types of instruments. In the Consolidated mass spectrometer the spectrum is covered by varying the ion accelerating voltage with constant magnetic field, and heavy ions are collected with less efficiency than light ions. This

1 Figures in brackets indicate the literature references at the end of this paper. introduces a small but unknown uncertainty in deriving total ionization by adding the mass peaks.

A greater source of error in published data comes from the determination of the sensitivity or current per unit pressure in the gas reservoirs. The common procedure for measuring this pressure has been to admit the gas into a volume of about 2 cubic centimeters and measure the pressure of 10 to 40 millimeters on a mercury manometer. Then the gas is expanded a thousandfold to give a pressure of 10 to 40 microns in the reservoir. The gas pipet method leads to very large errors when the vapor pressure is nearly equal to the manometer pressure and the estimated pressure will be much too high. Dibeler, Cordero, and Greenough [2, 3] have developed a micromanometer suitable for measuring directly the pressure in the gas reservoir. The pressure-sensitive element is a thin metal diaphragm and the motion of the diaphragm is measured electrically by the change in mutual induction between two coils close to the diaphragm.

Comparative measurements with the gas pipet and with the micromanometer show that use of the gas pipet leads to large experimental errors for all hydrocarbons with eight or more carbon atoms. For this reason all tables of mass spectra published by 